\title{
Percepção dos usuários de um centro de atenção psicossocial sobre seu projeto
}

\section{terapêutico singular}

\author{
Perception of users of a psychosocial care center about their unique therapeutic project \\ Percepción de los usuarios de un centro de atención psicosocial sobre su proyecto terapéutico único
}

Recebido: 03/08/2021 | Revisado: 12/08/2021 | Aceito: 16/08/2021 | Publicado: 18/08/2021

Iolanda Valéria Fernandes Tavares
ORCID: https://orcid.org/0000-0003-4263-6149
Escola de Saúde Pública, Brasil.
E-mail: iolandavaleria@hotmail.com
Janaína Quinzen Willrich
ORCID: https://orcid.org/0000-0001-7427-9305
Universidade Federal de Pelotas, Brasil
E-mail: janainaqwill@yahoo.com.br
Dariane Lima Portela
ORCID: https://orcid.org/0000-0003-1409-1823
Universidade Federal de Pelotas, Brasil
E-mail: dariane.lportela@hotmail.com

\begin{abstract}
Resumo
A partir da Reforma Psiquiátrica ocorrida no Brasil na década de 1970 houve uma reorientação do modelo de atenção psiquiátrica para um modelo de atenção psicossocial por meio dos Centros de Atenção Psicossociais (CAPS). Nesses espaços leva-se em consideração a individualidade dos usuários, possibilitando que junto da equipe se construa a sua trajetória de reabilitação psicossocial. O Projeto Terapêutico Singular (PTS) é uma das ferramentas utilizadas e consiste em um conjunto de propostas resultante da discussão coletiva entre equipe interdisciplinar, sujeito e família $\mathrm{O}$ presente estudo objetivou conhecer a percepção dos usuários de um CAPS acerca da construção e resolubilidade do seu PTS; identificar a resolubilidade das atividades terapêuticas e discutir a participação do usuário e familiares na elaboração do PTS. Trata-se de um estudo qualitativo exploratório, realizado em um CAPS do tipo II em Pelotas no Rio Grande do Sul. A pesquisa teve um total de dez participantes, utilizou-se entrevista semiestruturada e foi realizada durante o mês de setembro e outubro de 2019. A análise dos dados ocorreu por meio da análise temática.
\end{abstract}

Palavras-chave: Enfermagem; Saúde mental; Serviços de saúde mental; Sistemas de apoio psicossocial.

\begin{abstract}
Since the Psychiatric Reform that took place in Brazil in the 1970s there has been a reorientation of the model of psychiatric attention to a model of psychosocial attention through the Centers for Psychosocial Attention (CAPS). In these spaces the individuality of the users is taken into account, allowing the team to build their trajectory of psychosocial rehabilitation. The Singular Therapeutic Project (PTS) is one of the tools used and consists of a set of proposals resulting from the collective discussion between the interdisciplinary team, subject and family. The present study aimed at knowing the perception of users of a CAPS about the construction and resolubility of their PTS; identify the resolubility of therapeutic activities and discuss the participation of the user and family members in the elaboration of the PTS. It is a qualitative exploratory study, carried out in a CAPS type II in Pelotas, Rio Grande do Sul. The research had a total of ten participants, used semi-structured interview and was conducted during September and October 2019. The data analysis took place through thematic analysis.
\end{abstract}

Keywords: Nursing; Mental health; Mental health services; Psychosocial support systems.

\section{Resumen}

Desde la Reforma Psiquiátrica que tuvo lugar en el Brasil en el decenio de 1970 se ha producido una reorientación del modelo de atención psiquiátrica hacia un modelo de atención psicosocial a través de los Centros de Atención Psicosocial (CAPS). En estos espacios se tiene en cuenta la individualidad de los usuarios, lo que permite al equipo construir su trayectoria de rehabilitación psicosocial. El Proyecto Terapéutico Singular (PTS) es una de las herramientas utilizadas y consiste en un conjunto de propuestas resultantes de la discusión colectiva entre el equipo interdisciplinario, el sujeto y la familia. El presente estudio tiene como objetivo conocer la percepción de los usuarios de un CAPS sobre la construcción y resolubilidad de su PTS; identificar la resolubilidad de las actividades terapéuticas y discutir la participación del usuario y los miembros de la familia en la elaboración del PTS. Se trata de un estudio exploratorio cualitativo, realizado en un CAPS tipo II en Pelotas, Rio Grande do Sul. La investigación tuvo un total de diez participantes, utilizó una entrevista semiestructurada y se llevó a cabo durante septiembre y octubre de 2019. El análisis de los datos se realizó a través del análisis temático.

Palabras clave: Enfermería; Salud mental; Servicios de salud mental; Sistemas de apoyo psicossocial. 


\section{Introdução}

A Reforma Psiquiátrica teve início no Brasil mais especificamente no ano de 1978, quando surgiu um movimento criado por trabalhadores integrantes do movimento sanitário, sindicalistas, membros de associações de profissionais, associações de familiares e pessoas com históricos de internações psiquiátricas. A crítica ao saber psiquiátrico da época é inspiradora e alguns anos mais tarde em 1987 foi realizada a I Conferência Nacional de Saúde Mental no Rio de Janeiro. Também nesse ano na cidade de São Paulo foi criado o primeiro Centro de Atenção Psicossocial (CAPS) no Brasil (Brasil, 2005).

O CAPS é um serviço constituído por uma equipe multiprofissional que realiza atendimento às pessoas com transtornos mentais graves e persistentes, também às pessoas com necessidades decorrentes do uso de crack, álcool e outras drogas. As atividades no serviço são realizadas geralmente em espaços coletivos, onde os usuários participam de grupos, assembleias, etc. (Brasil, 2011). É considerado um serviço de portas abertas e sua equipe multidisciplinar juntamente com o usuário e seus familiares, planejam o Projeto Terapêutico Singular (PTS). É o único serviço que por meio das redes de apoio facilita e permite a reabilitação psicossocial de pessoas que pelo seu sofrimento, condição social ou descrédito, estejam vivendo excluídas da sociedade (Medeiros et al. 2016).

O PTS consiste em um conjunto de propostas e terapêuticas articuladas e que resulta de uma discussão coletiva entre equipe interdisciplinar, sujeito ou coletividade e família (Brasil, 2010). O PTS nos serviços de saúde mental é um meio de proporcionar uma atuação integrada a partir de aspectos relevantes acrescido do diagnóstico psiquiátrico e da medicação em uso. Seu conceito remonta ideias propostas a partir da reforma psiquiátrica, tendo como foco um modo de fazer que respeite a singularidade do sujeito e pressuponha maior articulação entre os membros da equipe utilizando as reuniões em equipe como um espaço coletivo para discussão, compartilhamento e corresponsabilização das ações (Brasil, 2013).

Dessa forma, o PTS consiste em uma ferramenta de trabalho única construída, coletiva e exclusivamente, para um indivíduo. E é através das suas histórias, vivências e níveis de entendimentos que o mesmo é construído (Brasil, 2011). A realização do PTS se dá através de três passos: identificar como se deu o início da relação (quais circunstâncias que o usuário procurou o serviço e qual sua trajetória de vida anterior); Realizar a cartografia da vida do usuário e seu contexto (entender sua história, sua trajetória de vida, suas relações com outras pessoas, suas fragilidades, possibilidades e potencialidades); e Criar um mapa multidimensional da vida (que deve conter detalhes das condições financeiras e materiais do usuário, assim como vulnerabilidades e riscos) (Brasil, 2005).

O presente estudo teve como objetivo conhecer a percepção dos usuários de um Centro de Atenção Psicossocial acerca da construção e resolubilidade do seu Plano Terapêutico Singular; identificar a resolubilidade das atividades terapêuticas indicadas e discutir a participação do usuário e familiares na elaboração do Plano Terapêutico Singular.

\section{Metodologia}

Esta pesquisa possui uma abordagem qualitativa exploratória, que identificou a percepção dos usuários a respeito do PTS proposto para o acompanhamento em um Centro de Atenção Psicossocial. De acordo com Minayo (2010), a pesquisa qualitativa dispensa números e estatísticas e apesar de abrir nossos olhos para novas fronteiras, não sabemos ao certo o que poderemos ver.

O estudo foi realizado em um Centro de Atenção Psicossocial do tipo II, na cidade de Pelotas, Rio Grande do Sul. O serviço se caracteriza como do tipo II, pois faz parte de um município ou região que atende mais de 70mil habitantes (Brasil, 2019). E contou com um total de dez participantes os quais respeitaram os seguintes critérios de inclusão: Ser usuário ativo no serviço há pelo menos três meses; concordar com o uso do gravador; e critérios de exclusão: exteriorizar condições emocionais e/ou cognitivas impeditivas para responder a entrevista. Os participantes foram convidados para participar do estudo e após o 
aceite assinaram em duas vias o Termo de Consentimento Livre e Esclarecido. Sua identificação nesse estudo se dá pela letra X, seguido do número 1, 2, 3, etc. Exemplo: Usuário X1 (primeiro participante da pesquisa), usuário X2 (segundo usuário da pesquisa) e assim sucessivamente até o usuário X10.

Foram respeitados os aspectos éticos conforme a resolução no 466 de 12 de dezembro de 2012 (Brasil, 2012), a qual aprova normas e diretrizes sobre pesquisas envolvendo seres humanos. A resolução traz como referenciais básicos da bioética: a autonomia, não maleficência, beneficência e justiça e ainda tende a assegurar os direitos e deveres que se remetem a comunidade cientifica, aos indivíduos da pesquisa e também ao Estado. Além de seguir o novo Código de Ética dos Profissionais de Enfermagem, resolução nº 564/17 (Conselho Federal de Enfermagem, 2017).

A aprovação pelo Comitê de Ética e Pesquisa se deu sob n.3.534.355. E ressalta-se que o estudo não refletiu em riscos físicos, pois não foi realizado nenhum procedimento doloroso ou coleta de material biológico, ou experimento com seres humanos. Os usuários tiveram como benefícios por sua participação a troca de conhecimentos, a reflexão quanto ao tratamento oferecido e a possível melhora no serviço futuramente.

Os dados para essa pesquisa foram coletados por meio da gravação de entrevista semiestruturada, que tiveram duração de aproximadamente 1 hora com cada participante. Foi realizada em uma sala privativa no CAPS onde os usuários realizam tratamento, durante o mês de setembro e outubro de 2019.

Para esse estudo foi utilizado a proposta de análise de dados temática de Minayo (2010), a qual após a leitura compreensiva dos textos oriundos das gravações das entrevistas, organiza-os pelas temáticas comuns, resultando em três categorias: resolubilidade, elaboração e sugestões.

\section{Resultados}

\section{Resolubilidade}

A partir da criação do projeto terapêutico singular os usuários são inseridos em atividades no serviço de saúde mental, as quais contribuem para o desenvolvimento da sua reabilitação e melhora de se quadro. Assim, entende-se que é importante que os mesmos avaliem a resolubilidade do PTS em sua trajetória de vida.

Durante as entrevistas os usuários relataram as atividades das quais participavam no CAPS, entre elas, oficinas terapêuticas, grupos terapêuticos, atendimentos individuais com médico, psicólogo e enfermeiros.

A partir desse contexto, todos os dez participantes informaram que de alguma maneira as atividades propostas e consultas médicas, os ajudam no dia-a-dia. Conforme podemos constatar na falas dos participantes:

"Ajudam e muito. Ajuda muito. Até acho que não existe lugar melhor que aqui" (X1).

"tive um contato com pessoas e isso já me ajudou bastante na questão de socialização. Esse lance de, de ter oura pessoa me observando, ajuda." (X4).

Ademais, podemos constatar que as atividades do serviço auxiliam inclusive no fomento à suas atividades de vida diária

[...] "Ah me ajudam bastante, se não fosse as oficinas eu não taria a, taria bem, não teria calma, não teria, é uma atividade que eu tenho que fazer né, se eu não faço nada em casa, eu faço só é o dever de casa né, lavar a roupa, fazer as coisas, limpar o pátio, varrer o quarto, ai aqui me ajuda, eu canto, eu exponho meus sentimentos, literatura e sentimentos, nós fazemos uma pergunta, em cima da pergunta nós fizemos uma resposta." (X7). 
Observa-se que os entrevistados percebem benefícios com o tratamento no serviço de saúde mental e demonstram satisfação com a assistência prestada pelos profissionais. Conforme o relato do usuário X4, que fala sobre conseguir socializar após o início do tratamento percebe-se que o trabalho realizado pelas equipes multidisciplinares no CAPS, contribui para a inclusão dos usuários na sociedade.

\section{Elaboração}

Outro critério relevante da construção do Projeto Terapêutico Singular consiste na elaboração do mesmo pela equipe multidisciplinar com a participação do usuário e familiares, a fim de encontrar atividades que possam ser a terapêutica mais adequada e possível para cada situação.

Quatro usuários informaram que nem eles e nem os familiares ajudaram a escolher as atividades em que estão inseridos, os outros seis informaram que das opções apresentadas pela equipe, tiveram a oportunidade de escolher onde melhor ficariam inseridos.

[...] "As gurias que indicaram, fiz o acolhimento e ai pelo meu perfil, enfim, pela conversa que a gente teve, eu cheguei em um momento bem delicado assim, bem em crise, eu já tava desassistida a alguns meses, em um momento peculiar da minha vida de uma frustração muito grande enfim, e ai elas fizeram o projeto e me apresentaram as possibilidades, e eu topei, vamos ver como é que a gente sai e ai deu super bem" (X10).

Percebe-se ainda certa dependência dos usuários em relação aos profissionais do serviço, demonstrando que ainda existe dificuldades em se romper a barreira do profissional aquele que determina algo, e o paciente aquele que cumpre. Como se percebe nas falas a seguir:

"Não. Eles que escolheram... A equipe do CAPS que escolhe as atividades" (X8).

"Não, não. Eu sempre participei das que eles escolheram pra mim. Eu nunca escolhi nada, o que eles escolhem pra mim tá bom." (X1).

Outrossim, alguns relatos nos demonstram que há certa negociação e oferta dos profissionais acerca de algumas atividades do serviço, como vemos na fala abaixo:

"Eu que pedi pra eles, pra fazer exercícios físicos né, por que eu gosto muito e, o grupo de mulheres foi indicado"(X6).

"Eu que das opções que tinham, eu escolhi" (X4).

"Perguntaram se queria, ai eu escolhi, eles ofereceram as oficinas, ai eu fui" (X7).

De acordo com os relatos, percebe-se que são expostos pela equipe as opções de atividades oferecidas pelo serviço e que é considerado também o desejo dos usuários para a realização de determinadas atividades e dessa forma é construído o Projeto Terapêutico Singular. E percebe-se que, nessa construção, a maioria dos usuários teve a oportunidade de participar, escolhendo as atividades em que estão inseridos.

\section{Sugestões}

Sobre a possibilidade de sugerir atividades em seu PTS, a metade dos entrevistados disse não possuir sugestões de 
mudanças, que da maneira como estava funcionando sentiam-se satisfeitos. O restante sugeriu mais acesso a consultas com a psicóloga, o retorno da terapeuta ocupacional e criação de mais oficinas e grupos, principalmente algum que abordasse mais sobre os ouvidores de vozes, conforme relatos que seguem:

"É, a sugestão é que um dia o Estado, tenha dinheiro pra bancar psicólogos, (risadas)!" (X4).

[...] "Nossa me relaxava muito, eu dormia muito bem de noite, e ai eu acho que deveria voltar né, outro projeto de terapia ocupacional muito bom. Eu tô sentindo um monte falta. (risos)." (X5).

[...] "Não, até então, não tenho nada a reclamar. A única coisa que eu, que no caso, gostaria que fosse mais seguido a psicóloga, que às vezes a gente precisa." (X6).

[...] "Se eu tenho alguma sugestão? Não pra mim tá especial, pra mim tudo, tudo que eles fazem aqui, tudo os projetos que eles fazem aqui, pra mim tá bom, tudo ta bom porque me faz bem." (X1).

Percebe também que, por vezes, não há nenhuma sugestão especifica, mas apenas anseios em participar e fazer diversas atividades

[...] "Eu queria fazer mais coisa, queria participar mais, participar mais de mais coisa. Mais projeto, eu queria, participar de projeto na rua, fazer um panelaço, que nem fazem na Argentina, divulgar a saúde mental que tá defasada né, que eles querem tirar a verba que não vem, a verba pra ajudar a prefeitura corta a verba, ta cada vez cortando mais a verba né, então eu queria fazer uma coisa pra ajudar sobre isso e falar sobre ouvidores de vozes, o que que é a ouvidoria de vozes, o que que é ver vultos, ouvir vozes, falar sobre essas coisas, eu queria ter uma coisa mais aprofundada sobre isso, que fosse mais divulgando." (X7).

A partir do exposto, parte dos usuários desta pesquisa percebe que o seu Projeto Terapêutico Singular é efetivo e sentem-se satisfeitos. Os que propuseram mudanças gostariam de mais profissionais na equipe para ampliar as consultas com especialistas psicólogos e terapeutas ocupacionais.

Assim, percebe-se que um maior número de profissionais na equipe multiprofissional poderia incrementar na diversidade de grupos e oficinas terapêuticas, possibilitando mais opções a serem desenvolvidas nos PTS dos usuários assistidos.

\section{Discussão}

Diante dos resultados deste estudo propomo-nos nessa seção discutir acerca dos estudos já publicados na área, a fim de problematizar os resultados encontrados, seja no intuito de corrobora-los ou refuta-los.

Para Carvalho et al., (2012) o Projeto Terapêutico Singular possibilita a participação de usuários e familiares no tratamento, a reinserção na sociedade e também a construção de autonomia.

Segundo Jorge et al., (2014) a resolubilidade do cuidado prestado em saúde mental requer a sistematização do serviço e o aperfeiçoamento das relações entre profissionais, usuários e familiares. O estudo mostra que visitas domiciliares, organização e realização de grupo terapêutico, atendimentos individuais dão continuidade no tratamento e representa uma perspectiva multiprofissional e interdisciplinar que favorece uma expectativa aumentada sobre as necessidades dos usuários no meio familiar, social e terapêutico, também promovendo a cidadania e assegurando que o usuário seja cuidado em suas necessidades biopsicossociais. 
Tais estudos vão ao encontro dos dados aqui relatados, quando os usuários relatam que visualizam resolubilidade no PTS, e ainda entendem que o mesmo auxilia no processo terapêutico de cuidado.

No estudo de Pinho, Souza e Esperidão (2018), familiares e usuários avaliam o CAPS positivamente o que evidencia que a proposta da Política Nacional de Saúde Mental, vem sendo bem executada. As vivências são relatadas como transformadoras e que assim, usuários podem viver de um modo autônomo e inclusivo na sociedade.

Isso nos faz refletir acerca da execução do PTS alinhada às políticas públicas possibilitar um maior protagonismo dos sujeitos envolvidos. Como pode-se ver nos dados aqui mencionados, a existência e inclusão de atividades multiprofissionais, como oficinas e grupos nos PTS's, potencializa a participação dos usuários e os valoriza enquanto agentes transformadores.

Para Ibiapina et al., (2017) principalmente as oficinas terapêuticas, proporcionam uma articulação com a política da reforma psiquiátrica, já que existe interesse para o trabalho por parte dos profissionais do CAPS e isso acarreta na transformação do indivíduo em sofrimento psíquico e sua readaptação na sociedade. A principal característica das oficinas terapêuticas é oferecer reflexão, conversas e construção de vínculos entre as pessoas, estimulando um resgate da sociabilidade e cidadania, além de respeitar o cuidado humanizado.

Kinker e Imbrizi (2015) acreditam que as oficinas terapêuticas articuladas no PTS consigam a transformação social necessária para o cotidiano. E que para haver essa transformação, o processo de mudança deve ser protagonizado pelos próprios usuários, deixando de serem "objetos" e partindo para ensinar a prática da construção de um mundo melhor.

Um estudo realizado por Pereira e Palma (2018), no CAPS da cidade de Unaí - Minas Gerais evidenciou que os usuários afirmam que as oficinas terapêuticas são um espaço onde podem manifestar seus sentimentos e pensamentos. Também é um espaço propício para o desenvolvimento de habilidades corporais e atividades produtivas. Alguns relatam ainda que o que aprendem em algumas oficinas artesanais pode ser utilizado como fonte de renda.

Segundo Cardoso, Oliveira e Piani (2016) a participação de usuários nos CAPS promove benefícios em sua terapêutica. Conseguem desenvolver habilidades, melhorar a autoestima, promover a autonomia, exercer cidadania, autoconhecimento, lazer, discutir questões do dia a dia, discutir sobre medicações e ainda possibilitar a integração dos familiares ao tratamento.

Outrossim é importante atentar para o quê o estudo realizado por Silva, Lima e Ruas (2018), realizado em diversos CAPS de Minas Gerais nos traz, que os usuários em sua grande maioria estavam satisfeitos com o atendimento prestado pelos profissionais, assim como em outros lugares do país. Mas que é importante considerar que altos índices de satisfação, podem ocorrer devido a familiaridade do usuário com o serviço, sua gratidão por melhoras e também à valorização por estarem sendo assistidos fora de um modelo manicomial.

E nesse sentido entendemos que por estarem dentro de um contexto de reabilitação psicossocial, um modelo substitutivo às práticas que foram tão excludentes e adoecedoras no histórico de saúde mental pode haver esse tipo de associação do entrevistado. Bem como a existência de condutas que aceitam o que o profissional decide em seu PTS, como também visualizamos nas falas da seção anterior. Entendemos que não é essa perspectiva que intenciona o projeto terapêutico, mas sim uma construção mútua, entre profissional e usuário.

Silva (2016), nos sinaliza que o PTS é uma ferramenta eficaz e é necessário que a sua criação advenha de um modelo organizado pela equipe de saúde, onde devem ser rígidos através dos protocolos específicos, no entanto, deve levar em consideração as vontades do sujeito. O PTS é uma tática que sistematiza o cuidado e que se constrói de forma colaborativa com o usuário, sua família, e equipe do serviço.

Pouco se observa nas falas deste estudo a participação do usuário e famílias nas decisões acerca do PTS. Entende-se que politicamente é o necessário, e correto a ser feito. Mas na prática ainda se enfrenta bastante distanciamento entre profissional e usuário, fazendo parecer que ainda estamos diante de práticas prescritivas. 
Para Covelo e Moreira (2015), há necessidade de voltar o olhar para os familiares a fim de perceber a problemática familiar em que o usuário está inserido, assim a participação dos mesmos pode ser eficaz na elaboração do Projeto Terapêutico Singular. Essa participação no cuidado prestado às pessoas em sofrimento psíquico é fundamental na atenção psicossocial, pois a mesma é um meio de constante mudança de ligações afetivas e sociais e o campo da saúde mental tem o desafio contínuo de aproximação.

Grigolo et al. (2015) enfatizam que o PTS é um Projeto, pois se trata de uma construção inacabada, aberta a provisões e mudanças. Terapêutico, pois vê o cuidado como uma construção nas práticas de saúde. Singular, porque se refere ao usuário e sua família. O mesmo prevê uma prática participativa, integrada e compartilhada entre o usuário, seu técnico de referência e demais profissionais do serviço. O PTS é um dispositivo potente, já que possibilita a ampliação da gestão do cuidado, num exercício compartilhado, permitindo a dimensão inventiva e criativa dos caminhos e produções para o cuidado contando com uma equipe multidisciplinar.

É nessa perspectiva de construção que se acredita ser necessário maior saliência dos usuários em sua terapêutica, o que nos parece carecer ainda envoltos em uma prática bastante cultural em que o indivíduo sofredor de um transtorno mental se dá por satisfeito somente pelo fato de não mais estar no ambiente hospitalar.

Ibiapina et al (2017), fortalecem a perspectiva de que para buscar um suporte terapêutico aos usuários, deve-se contar com o apoio da família e comunidade. E a diversidade das atividades realizadas é de suma importância para o acolhimento integral do usuário, assim o mesmo pode interagir e verificar a melhor maneira de dar continuidade no seu tratamento.

Segundo Silva, Lima e Ruas (2018), o contentamento dos usuários é um importante critério para mensurar a qualidade dos Centros de Atenção Psicossocial. O estudo dos autores revelou que usuários de onze CAPS do Estado de Minas Gerais, além de satisfeitos com o serviço, contribuíam com suas sugestões para avaliação do mesmo. Ademais, usar o ponto de vista dos usuários como critério de avaliação é uma mudança no padrão da saúde mental, já que, historicamente, a pouco tempo subestimavam sua participação. A prática dos serviços prestados necessita da aceitação dos usuários e também do cumprimento de critérios técnicos.

De acordo com o estudo de Bittencourt et al., (2018) em um CAPS da cidade de Macapá, percebeu como resultado que os usuários estavam satisfeitos com o atendimento multidisciplinar da equipe, porém, reclamavam a falta de opções e novas formas de oficinas terapêuticas.

Para Cardoso, Oliveira e Piani (2016) para haver uma melhora na assistência prestada nos CAPS é necessário o aumento do número de profissionais, principalmente de médicos para suprirem a grande demanda, embora a necessidade de um maior número de médicos não tenha sido apontada nesta pesquisa.

Isso é um ponto que sempre pode recrudescer as práticas psicossociais, a oferta de mais profissionais e qualificados para atender a demanda em saúde mental pode possibilitar um maior fortalecimento das atividades oferecidas pelo serviço, o que pode refletir em mais possibilidades na construção do PTS.

O que vai ao encontro das falas em que os usuários trazem sugestões de práticas que poderiam estar presentes nos serviços, e que não mais estão disponíveis. Percebe-se que eles sentem faltas de inúmeras atividades, que talvez num contexto de melhor organização política e profissional, pudesse estar sendo oferecido.

\section{Considerações Finais}

Com esse estudo destacaram-se pontos centrais na percepção dos usuários de um centro de atenção psicossocial sobre seu projeto terapêutico singular. Os usuários identificam a resolubilidade do PTS em seu processo terapêutico, no entanto nem sempre fazem parte, junto de seus familiares, da elaboração do mesmo. Percebe-se que há sugestão de atividades por parte dos participantes, as quais poderiam potencializar o seu PTS. 
Este estudo traz uma discussão alinhada ao cuidado psicossocial, atentando para o protagonismo dos usuários a respeito do seu envolvimento na criação do Projeto Terapêutico Singular. Nos demonstra que, enquanto profissionais, serviços ou instituições, precisamos estar abertos e voltados para o envolvimento dos mesmos em seu processo de cuidado, pois isso é saudável e proporciona uma melhor relação e vínculo de serviços e usuários.

\section{Referências}

Bittencourt, M. N., Oliveira, D. C. P., Souza, R. P. O., PenaI, J. L. C., PantojaI, P. V. N., \& Pereira, M. O. (2018). A Experiência da Ouvidoria em um Centro de Atenção Psicossocial Álcool/Drogas. Revista Brasileira de Enfermagem. 71(5), 2421-2428. https://doi.org/10.1590/0034-7167-2017-0924

Brasil. (2019). Cadastro Nacional de Estabelecimentos de Saúde. http://cnes.datasus.gov.br/pages/estabelecimentos/ficha/identificacao/4314403042952

Brasil. (2016). Decreto $N^{o} 510$ de 7 de abril de 2016. http://conselho.saude.gov.br/resolucoes/2016/Reso510.pdf

Brasil. (2005). Reforma Psiquiátrica e Política de Saúde Mental no Brasil. http://bvsms.saude.gov.br/bvs/publicacoes/Relatorio15_anos_Caracas.pdf

Brasil. (2006). Política Nacional de Humanização. http://bvsms.saude.gov.br/bvs/publicacoes/documento_base.pdf

Brasil. (2011). Portaria No 3.088 de 23 de Dezembro de 2011. http://bvsms.saude.gov.br/bvs/saudelegis/gm/2011/prt3088_23_12_2011_rep.html

Brasil. (2012). Resolução 466 de 12 de dezembro de 2012. http://conselho.saude.gov.br/resolucoes/2012/Reso466.pdf

Cardoso, M. R. O., Oliveira, P. T. R., \& Piani, P. P. F. (2016). Práticas de cuidado em saúde mental na voz dos usuários de um Centro de Atenção Psicossocial do Estado do Pará. Revista saúde e Debate. 40 (109), 86-99. http://dx.doi.org/10.1590/0103-1104201610907

Conselho Federal de Enfermagem. (2017). Novo Código de Ética dos Profissionais de Enfermagem. http://www.cofen.gov.br/resolucao-cofen-no5642017_59145.html

Covelo, B. S. R., \& Moreira, M. I. B. (2015). Laços entre família e serviços de saúde mental: a participação dos familiares no cuidado do sofrimento psíquico. Revista Interface.19 (55), 1133-1144. http://dx.doi.org/10.1590/1807-57622014.0472

Grigolo, T. M., Garcia, C. A. S., Peres, G. M., \& Rodrigues, J. (2015). O projeto terapêutico singular na clínica da atenção psicossocial. Cadernos brasileiros de Saúde Mental. 7(15), 53-73.

Ibiapina, A. R. S., Monteiro, C. F. S., Alencar, D. C., Fernandes, M. A., \& Filho, A. A. I. C. (2017). Oficinas Terapêuticas e as mudanças sociais em pacientes com transtorno mental. Escola Anna Nery. 21 (3), 1-8. https://doi.org/10.1590/2177-9465-ean-2016-0375

Jorge, M. S. B., et al (2014). Resolubilidade do cuidado em saúde mental na estratégia de saúde da família: Representação social de profissionais e usuários. Revista Escola de Enfermagem USP. 48 (6), 1062-1068. https://doi.org/10.1590/S0080-623420140000700014

Kinker, F. S, \& Imbrizi, J. M. (2015). O mito das oficinas terapêuticas. Revista Polis e Psique. 5 (3), 61-79.

Medeiros, P. F. P., et al 2017) Rede de Atenção Psicossocial no Sistema Único de Saúde - Eixo Políticas e Fundamentos. http://www.aberta.senad.gov.br/medias/original/201704/20170424-094953-001.pdf

Minayo, M. C. S. (2010). O desafio do conhecimento: Pesquisa qualitativa em saúde (12 ed.). Editora Hucitec.

Pereira, O. P., \& Palma, A. C. R. (2018). Sentido das oficinas terapêuticas ocupacionais do CAPS no cotidiano dos usuários: uma descrição fenomenológica. Revista da Abordagem Gestáltica. Goiânia. 24 (1), 15-23. http://dx.doi.org/10.18065/RAG.2018v24n1.2

Pinho, E. S., Souza, A. C. S., \& Esperidão, E. (2018). Processos de Trabalho dos Profissionais dos Centros de Atenção Psicossocial: revisão integrativa. Ciência e Saúde Coletiva. 23 (1), 141-151. http://dx.doi.org/10.1590/1413-81232018231.08332015.

Silva, G. S. (2016). A construção do projeto terapêutico singular em saúde mental para "o senhor moeda" numa experiência de abordagem intersetorial. Revista de trabalhos acadêmicos - universo belo horizonte. 1 (1), 1-6.

Silva, S. N., Lima, M. G., \& Ruas, C. M. (2018). Avaliação de Serviços de Saúde Mental: Satisfação dos usuários e fatores associados. Ciências e Saúde Coletiva. 23 (11), 3799-3810. https://doi.org/10.1590/1413-812320182311.25722016 